\title{
Evaluating the Trends of Bloodstream Infections by Nonfermenting Gram Negative Bacilli among the Patients in a Tertiary Care Hospital of Western Part of India and its Antibiogram
}

\section{Nabamita Chaudhury ${ }^{1}$, Retina Paul ${ }^{2}$, R.N. Misra ${ }^{3}$, Sankha Subhra Chaudhuri ${ }^{*}$, Shazad Mirza ${ }^{3}$ and Sukanta Sen ${ }^{5}$}

${ }^{1}$ Department of Microbiology, Burdwan Medical College and Hospital, Purba Bardhaman, West Bengal, India

${ }^{2}$ Department of Microbiology, College of Medicine and JNM Hospital, Nadia, West Bengal, India

${ }^{3}$ Department of Microbiology, Dr. D.Y. Patil Medical College, Hospital and Research Centre, Pune, Maharashtra, India

${ }^{4}$ Department of Ophthalmology, Burdwan Medical College and Hospital, Purba Bardhaman, West Bengal, India

${ }^{5}$ Department of Pharmacology, ICARE Institute of Medical Sciences and Research, Banbishnupur, Purba Medinipur, Haldia, West Bengal, India

*Corresponding author

\section{Keywords \\ Gram-Negative \\ Non-Fermenting \\ Bacilli (NFGNB), \\ Blood Stream \\ Infections (BSIs), \\ Multi-drug \\ resistance}

\section{Article Info}

Accepted:

10 December 2018

Available Online:

10 January 2019

\section{A B S T R A C T}

Non-fermenting gram-negative bacilli (NFGNB) are an emerging problem in Blood stream infections. A major concern is multi-drug resistance which severely limits treatment options. Earlier it was believed to be non pathogenic' but recently they are more frequently isolated as primary pathogen. Usually they cause hospital acquired infection (HAI). A prospective study was conducted to isolate the NFGNB from blood samples, to identify the risk factors leading to blood stream infections and to determine the antibiotic susceptibility pattern of them. The study was conducted in a tertiary care hospital, over a period of 2 years. Identification of NFGNB was done by biochemical tests and by VITEK 2 . Antibiotic susceptibility was determined by disc diffusion method. Extended-spectrum $\beta$-lactamases (ESBLs) and metallo- $\beta$-lactamases (MBLs) production were detected by the combined disc diffusion test. Out of 2021 blood samples, blood culture positive was in $32.7 \%$ of patients of whom the cause was NFGNB. Acinetobacter boumannii was the most common organism, $27.69 \%$ followed by Strenotrophomonas maltophilia, next to it was Pseudomonas aeruginosa Acinetobacter lwoffiietc. The most common risk factors for colonization BSIs with NFGNB was comorbid conditions, such as diabetes mellitus, cardiovascular diseases, hypertension, tuberculosis and chronic renal disease patients on haemodialysis. In general, the isolates of NFGNB revealed pretty much good sensitivity to carbapenem (imipenem, ertepenam), colistin and aminoglycosides (amikacin, gentamicin), where as cephalosporin group revealed a low susceptibility rate. ESBL and MBL producer NFGNB were identified and the isolation rate is very alarming. The trend of increasing numbers of cases of NFGNB in Blood stream infections compounded by MDR is of great concern. It is necessary to administer antibiotics judiciously, strengthen surveillance and laboratory services in intensive care units, and re-evaluate treatment guidelines for management of infection by these organisms. 


\section{Introduction}

The non-fermenting organisms are comprised of gram negative rod shaped bacilli. ${ }^{1}$ The non fermenting gram negative bacilli (NFGNB) are taxonomically group of aerobic non spore forming bacilli that either do not utilize carbohydrates as the source of energy or degrade them through metabolic pathways other than fermentation. ${ }^{2}$ They are widely distributed in nature as saprophytes, found in soil, water, sewage or as commensals on human skin or in the human gut and some of them found in hospital environment. $1,3,4$ These nonfermenters are unfortunately the byproduct of medical and surgical advances in health care system of serious ill patients. ${ }^{5}$ Recently, these NFGNB are emerging problem in sepsis, which is associated with significant mortality and morbidity. A major concern is multi-drug resistance which severely limits treatment options.

The predominant species of concern among NFGNB are Pseudomonas aeruginosa, Acinetobacter baumannii, Strenotrophomonas maltophilia and, less so, members of the Burkholderia cepacia group. ${ }^{3}$ Except $P$. aeruginosa the NFGNB are most often cause nosocomial infections in immunecompromised patients like urinary tract infections (UTI), Bloodstream infections (BSIs), ventilator associated pneumonia (VAP) and surgical site infections (SSI). ${ }^{1}$

Bloodstream infections (BSIs) are the significant causes of morbidity and mortality for many patients. ${ }^{6}$ BSIs are defined as the presence of viable infectious microorganism in the bloodstream causing clinical illness. ${ }^{7}$ The term bloodstream infection and bacteremia are synonymously used, which generally refer to the significant growth of a microorganism in a blood culture obtained from the patient with clinical signs of infection. ${ }^{8}$ Bacteremia may range from self- limiting infections to septicaemia which is life threatening and needs rational antimicrobial treatment. ${ }^{9}$ In the developing countries, like India lack of standard antimicrobial guidelines, emergence of antimicrobial resistance, paucity of good diagnostic facilities and poor hospital environment, poor quality of hand hygiene are major denominators for surge in BSI associated morbidity and mortality. ${ }^{10}$

\section{Materials and Methods}

This was a prospective study. The study was conducted in the Microbiology Department of Dr. D.Y. Patil Medical College, Hospital and Research Centre, over a period of 2 years (i.e. July 2012 to September 2014). A total 2021 blood samples from the suspected patients of sepsis were collected in the adult and paediatric patients. Bloods were collected aseptically in brain heart infusion broth (BHI) or in BACT/ALERT 3D system. In case of neonates $2 \mathrm{ml}$ blood, children 3-5 ml blood and for the adults $10 \mathrm{ml}$ blood were taken. The samples were taken from the suspected patients, admitted to different wards and various intensive care units (ICU) of this hospital. The study was approved by the Ethical Committee of our institute.

Blood samples were processed for culture by standard conventional methods. Identification of Nonfermenters were carried out by Gram staining (gram negative bacilli/ gram negative coccobacilli), cell and colony morphology, pigment production, catalase test, $\mathrm{p}$ citrate test, triple sugar iron (alkaline slant/ no change butt), oxidase test and by motility test. Further identification was done by Hugh and Leifson oxidative-fermentative test (O-F) for glucose, sucrose, lactose, mannitol; gelatin liquefaction, nitrate reduction test, Decarboxylation of arginine, lysin and ornithine and growth at $35^{\circ} \mathrm{C}$ and at $42^{\circ} \mathrm{C}$ for 18-24 hours on two tubes of trypticase soy 
agar (TSA). The final identification and confirmation was done by the Vitek 2 system. $^{2}$

Identification of pigment production by King's A and King's B medium ${ }^{11}$

King's A medium ${ }^{11}$ : Pyocyanin, a blue phenazine derivative characteristic of $P$. aeruginosa was diffusible and its production was enhanced by growth in "King A (Fig. $1)^{\prime} .{ }^{11}$

\section{King's B medium ${ }^{11}$ : Fluorescent} Pseudomonas were characterised by production of water soluble pigment, which diffused freely in the media and fluoresce brightly under U.V ray. The organisms produced this pigments were $P$. aeruginosa, $P$. putida, $P$. fluorescens, $P$. chlororaphis etc. and was manifested in low iron containing media. 6 "King B" medium was the universally use medium for the production of fluorescent pigment. ${ }^{11}$

Antibiotic susceptibility testing was determined by Kirby - Bauer disc diffusion method $^{2,12}$

Muller-Hinton agar media was used. Commercially available Himedia discs were used. The strength of the discs used and their zone size interpretation were carried out by National Committee for Clinical Laboratory Studies (NCCLS) guideline. The antibiotics, which were tested, Piperacillin $(10 \mathrm{mcg} / \mathrm{disc})$, Carbenicillin (100mcg/disc), Ampicillin (10mcg/disc), Cefotaxim (30mcg/disc), Ceftriaxone (30 mcg/disc), Ceftazidime (30 mcg/disc), Cotrimaxazole ( $25 \mathrm{mcg} / \mathrm{disc})$, Ciprofloxacin (5 mcg/disc), Norfloxacin (10 $\mathrm{mcg} / \mathrm{disc}) \quad$ Gentamicin (10mcg/disc), Amikacin (30 mcg/disc), Imipenem (10mcg/disc), Chloramphenicol (30 mcg/disc) Tobramycin (10mcg/disc), Ofloxacin (5mcg/disc), Amoxicillin/Clavulanic acid
(20/10 mcg/disc), Piperacillin/Tazobactam (100/10 mcg/disc), Tigecycline (15mcg/disc), Colistin $(10 \mathrm{mcg} / \mathrm{disc})$ and Ertepenem (10mcg/disc).

\section{Detection of extended spectrum $\beta$ - lactamases production ${ }^{12,13}$}

The Combine disk diffusion test (CDDT) was used to determine the prevalence of extended spectrum $\beta$-lactamases (ESBL) production. Muller-Hinton agar media was used. One Ceftazidime (CAZ) $(30 \mu \mathrm{g})$ disc was placed on a lawn culture of test isolates and at the distance of $15 \mathrm{~mm}$ on both side of CAZ disc, a combination disc of Ceftazidime/ Tazobactam $(30 / 10 \mu \mathrm{g})$ and Ceftazidime / Clavulanic acid $(30 / 10 \mu \mathrm{g})$ were placed. $\mathrm{A} \geq 5$ $\mathrm{mm}$ increased in a zone diameter for either antimicrobial agent tested in combination with Clavulanic acid or Tazobactam versus the zone diameter of the agent when tested alone $=$ ESBL producer (Fig. 2) ${ }^{10,13}$

\section{Detection of metallo $\beta$-lactamases production}

Muller-Hinton agar media was used. One Imipenem $(10 \mu \mathrm{g})$ disc was placed on a lawn culture of isolates and at the distance of 15 $\mathrm{mm}$ a combination disc of $10 \mu \mathrm{g}$ of Imipenem and $100 \mu 1$ of EDTA disc was placed. Then it was incubated at $35^{\circ} \mathrm{C}$ for $18-24$ hours. An increase in zone size $\geq 7 \mathrm{~mm}$ around the Imipenem -EDTA disc as compared to Imipenem disc alone was recorded as positive (Fig. 3). ${ }^{10,13}$

\section{Results and Discussion}

In this study, out of 2021blood samples, total number of culture positive isolates were 661 (32.7\%) among which $445(67.32 \%)$ were gram positive cocci (GPC) and $216(32.68 \%)$ were gram negative bacilli (GNB). Out of 216 GNB, 65 (30.1\%) were non-fermenting gram 
negative bacilli (NFGNB). Out of the total 65 isolates, highest number of isolates (23\%) were obtained from male surgical ward, followed by Medicine Intensive Care Unit (MICU) (10.8\%) next to it was male medicine ward (9.6\%) (Fig. 4). While discussing about the gender distribution, in this study male $(69.23 \%)$ outnumbered the female $(30.77 \%)$ (Fig. 5). In our study the patients were divided into ten age groups. The majority of the patient belongs to 41 to 50 years, accounting for $27 \%$, followed by the age group of 31 to 40 years comprises $18 \%$, next to this is the age group of 11 to 20 years accounting for $9.23 \%$ (Fig. 6).

The highest number of isolates were Acinetobacter boumannii, comprises $27.69 \%$ followed by Strenotrophomonas maltophilia (previous designation: Pseudomonas maltophilia) 21.53\%, next to it was Pseudomonas aeruginosa (13.84\%), Acinetobacter lwoffii (6.15\%), Pseudomonas fluroscence (4.61\%), Acinetobacter boumannii complex (ABC) (4.61\%), Burkhelderia cepacia (4.61\%) (previous designation: Pseudomonas cepacia), Sphingomonas paucimobilis (3.07\%) (previous designation: Pseudomonas paucimobilis), Pseudomonas stutzeri (3.07\%), Pseudomonas putida (3.07\%) and each one isolates of Acinetobacter radioresistance, Acinetobacter calcoaceticus, Acinetobacter haemolyticus, Burkholderia multivorans and Moraxella oslonensis (Table 1).

In this study we have analyzed the risk factors for colonization BSIs with NFGNB. Prolonged hospitalization, mechanical ventilation, indwelling foreign devices (especially orthopedic implants, in-situcanula), unjudicial antimicrobial therapy and comorbidities, have identified as risk factors which are predisposing to acquisition BSIs by NFGNB. In this study $29.23 \%$ isolates were obtained from the patients who had comorbid conditions, such as diabetes mellitus, cardiovascular diseases, hypertension, tuberculosis and chronic renal disease patients on haemodialysis. Around $24.61 \%$ isolates were obtained from the patients, who were on indwelling intravascular catheters or orthopedics implants in situ, followed by $18.46 \%$ of isolates from those patients who have admitted in this hospital for a long tenure, next to it was $15.38 \%$ isolates from those patients who were on mechanical ventilators and $12.31 \%$ isolates were yield from the patients who had prolonged history of hospitalization (Fig. 7).

The isolates of Pseudomonas aeruginosa revealed $100 \%$ sensitivity to Colistin and also revealed good susceptibility to Ertepenam (90.8\%) followed by Imipenem $(86.77 \%)$, Tobramycin (66.66\%) next to it, was Amikacin (64.02\%) (Fig. 8). The isolates of Acinetobacters showed $60 \%$ were sensitive to Imipenem. In this study we have reported $52.2 \%$ susceptibility to chloramphenicol and $48.9 \%$ to gentamicin. Close to it, in this study amikacin and norfloxacin each comprises of 47.8\%. In this study Ceftazidime shows a bit low sensitivity pattern, accounting for $37.8 \%$ (Fig. 9).

The isolates of Strenotrophomonas maltophilia showed $100 \%$ sensitivity to Colistin revealed good susceptibility to Ertepenam (96.65\%), Ofloxacin (94.12\%), Ceftazidime (94.12\%) followed by Ciprofloxacin (88.23\%) (Fig. 10). Among the total 65 isolates of NFGNB, 20 isolates $(30.77 \%)$ were multidrug resistance (MDR). However, amidst these 20 isolates 11 (55\%) were ESBL- producers and rest (45\%) were MBL- producers.

S. maltophilia showed a good sensitivity to ertepenam (96.65\%), ofloxacin (94.12\%), ceftazidime $(94.12 \%)$ and ciprofloxacin $(88.23 \%)$ 
Table.1 Distribution of non-fermenting gram negative bacilli in different clinical samples $(n=65)$

\begin{tabular}{|l|l|}
\hline Name of the organism & Number of isolates (\%) \\
\hline Pseudomonas aeruginosa & $9(13.84 \%)$ \\
\hline Pseudomonas fluroscence & $3(4.61 \%)$ \\
\hline Pseudomonas putida & $2(3.07 \%)$ \\
\hline Pseudomonas stutzeri & $2(3.07 \%)$ \\
\hline Acinetobacter boumannii & $18(27.69 \%)$ \\
\hline Acinetobacter boumanniicomplex(ABC) & $\mathbf{3 ( 4 . 6 1 \% )}$ \\
\hline Acinetobacter lwoffii & $\mathbf{4 ( 6 . 1 5 \% )}$ \\
\hline Acinetobacterradioresistance & $1(1.53 \%)$ \\
\hline Acinetobactercalcoaceticus & $1(1.53 \%)$ \\
\hline Acinetobacter haemolyticus & $1(1.53 \%)$ \\
\hline Burkholderiacepacia & $3(4.61 \%)$ \\
\hline Burkholderiamultivorans & $1(1.53 \%)$ \\
\hline Strenotrophomonasmaltophilia & $14(21.53 \%)$ \\
\hline Sphingomonaspaucimobilis & $2(3.07 \%)$ \\
\hline Moraxella oslonensis & $1(1.53 \%)$ \\
\hline Total & $\mathbf{6 5}$ \\
\hline
\end{tabular}

Fig.1 Kings B mediumunder U-V ray

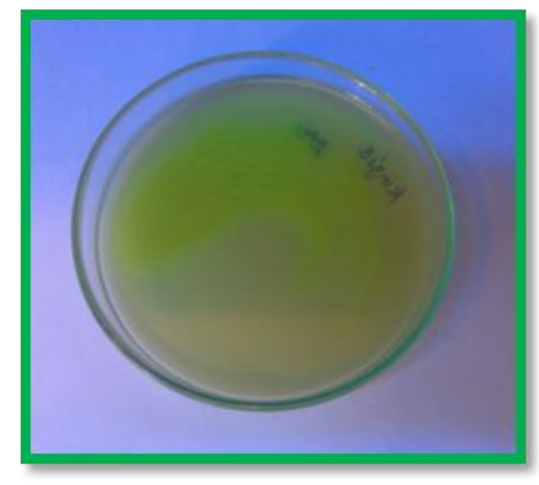

Fig.2 ESBL producer

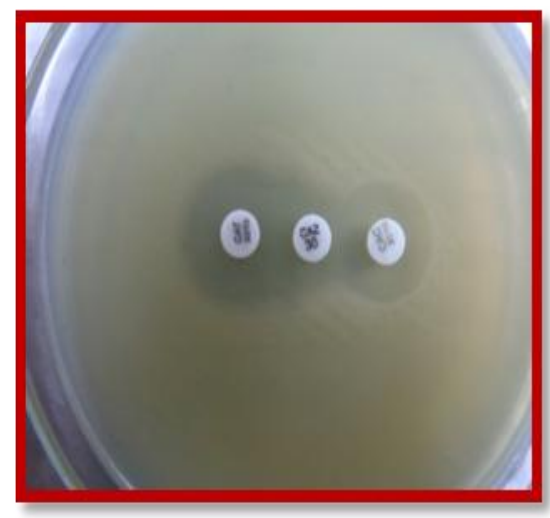


Fig.3 MBL producer

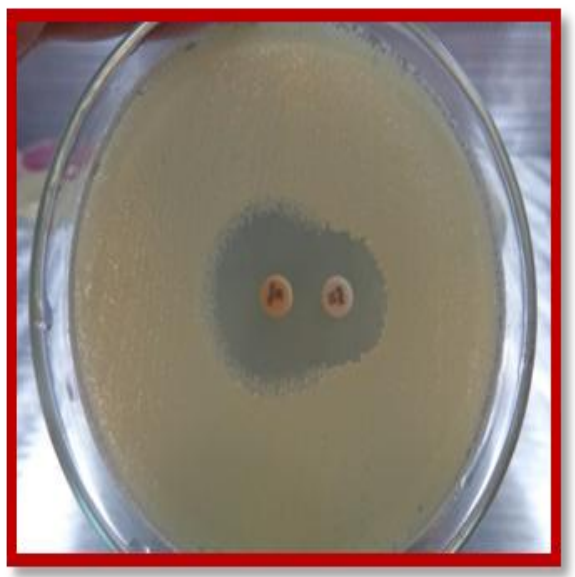

Fig.4 Ward wise distribution of different clinical samples $(n=65)$

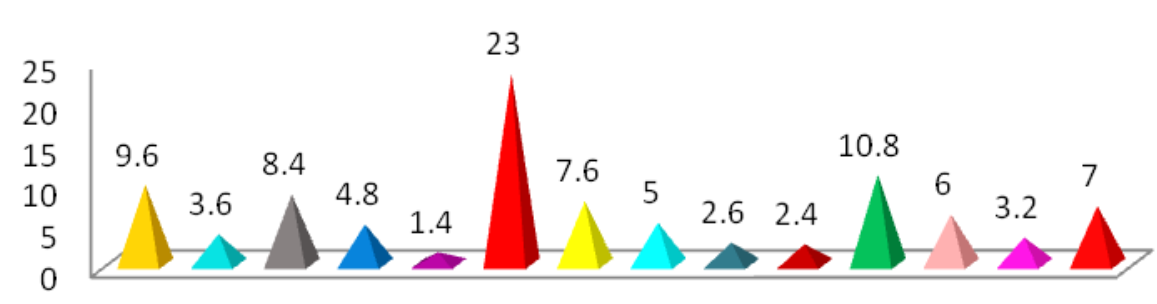

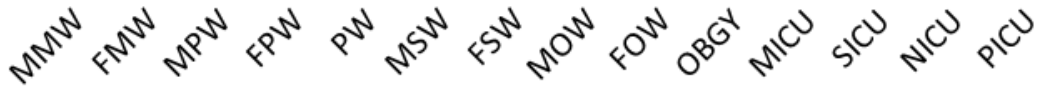

$\square \mathrm{MMW} \backsim \mathrm{FMW} \square \mathrm{MPW} \backsim \mathrm{FPW} \quad \mathrm{PW} \quad \mathrm{MSW} \backsim \mathrm{FSW}$

$\square \mathrm{MOW} \backsim \mathrm{FOW} \backsim \mathrm{OBGY} \square \mathrm{MICU} \backsim \mathrm{SICU} \square \mathrm{NICU} \square \mathrm{PICU}$

Fig.5 Gender distribution of the patients $(n=65)$

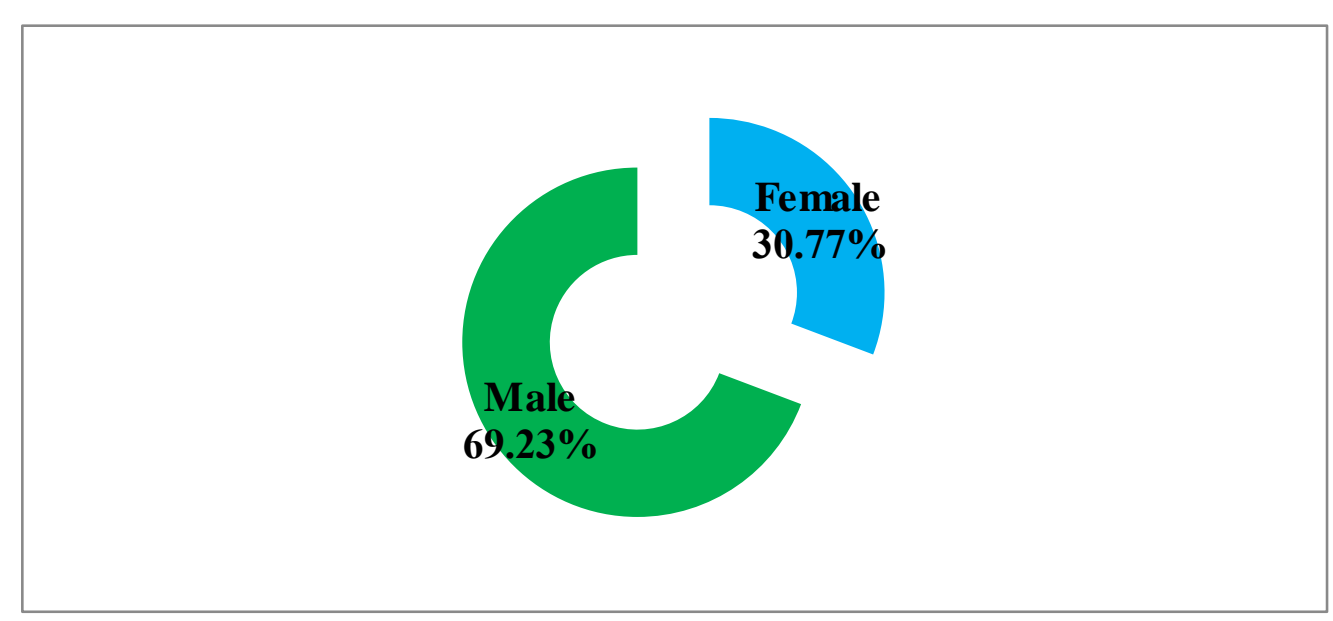


Fig.6 Age distribution of the patients $(n=65)$

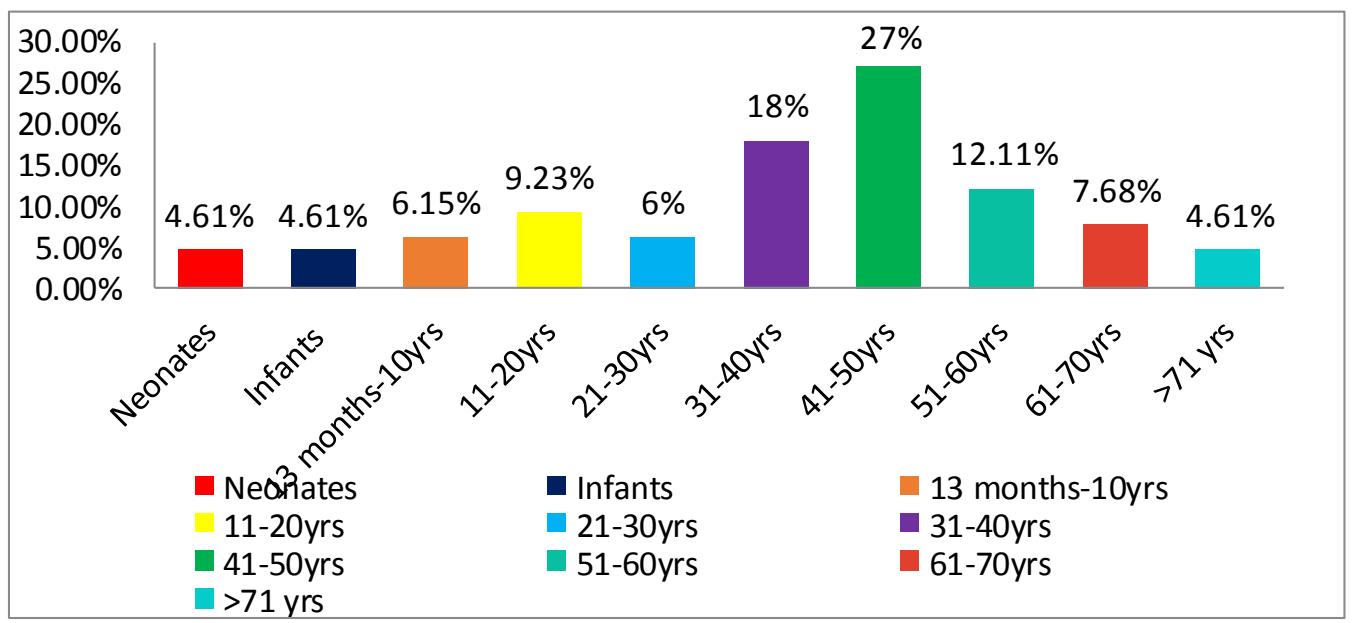

Fig.7 The incidence of infection due to gram negative nonfermenting organisms

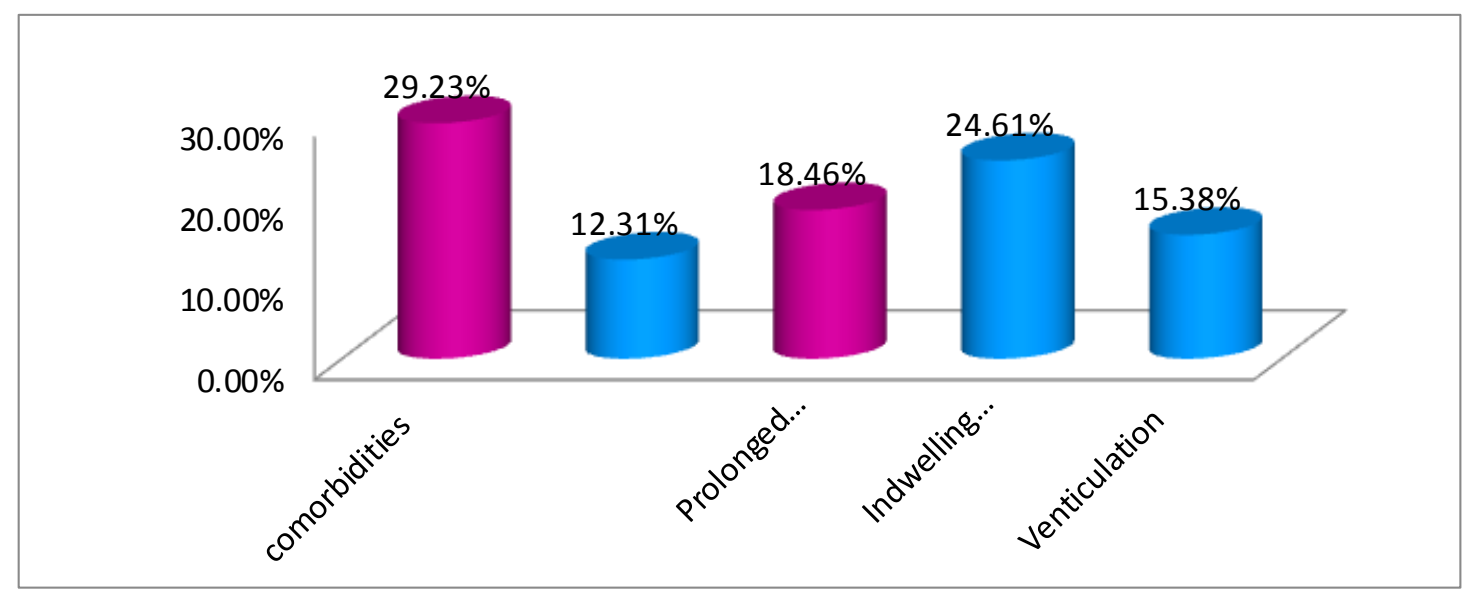

Fig.8 Antibiotic susceptibility pattern of Pseudomonas aeruginosa $(\mathrm{n}=9)$

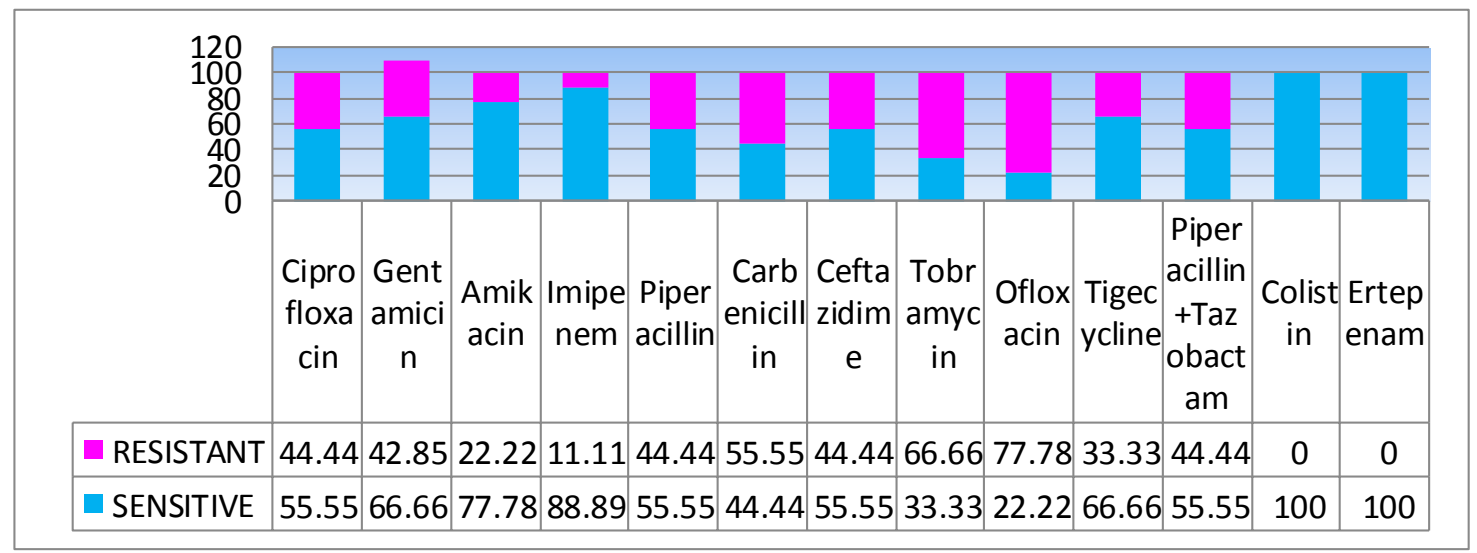


Fig.9 Antibiotic susceptibility pattern of Acinetobacter species $(\mathrm{n}=28)$

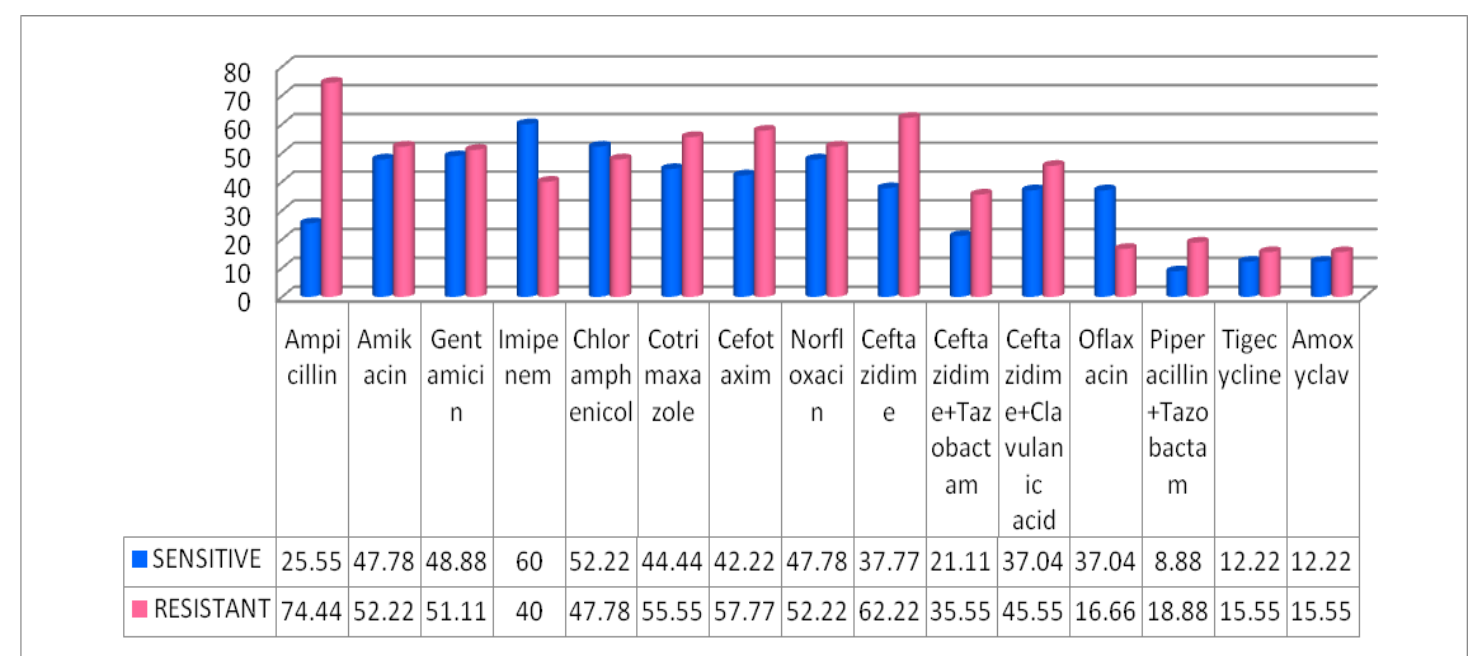

Fig.10 Antibiotic susceptibility pattern of Strenotrophomonas maltophilia $(\mathrm{n}=14)$

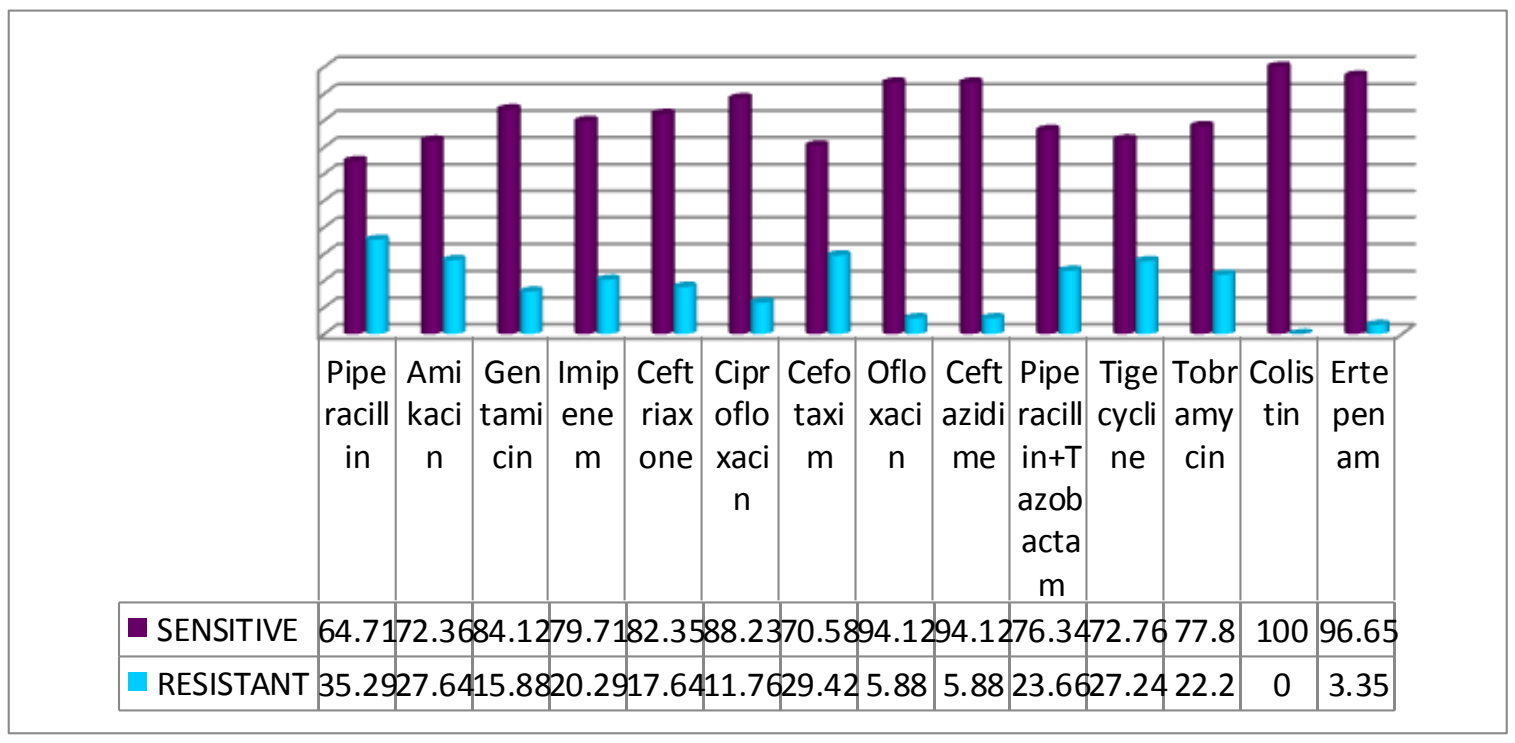

Bloodstream infections by NFGNB remained a challenge for the clinician and microbiologists due to the limited facilities in the laboratories to identify NFGNB, changing bacterial etiology and emergence of antimicrobial resistance. Early detection of NFGNB and determination of its antimicrobial susceptibility can reduce the occurrence of BSI and can also decrease the rate of emergence of MDR isolates. Our study evaluates the incidences of bloodstream infections by NFGNB, risk factors underlying and antimicrobial susceptibilities among the paediatric and adult group of patients.

The non-fermenting gram negative bacilli are found in nature as inhabitants of soil, water and also the commensals of human and animal mucous membranes. Recently these organisms are gaining importance as the frequently isolated primary pathogen in patients with prolonged hospitalization. 
NFGNB have the ability to adapt well in hospital environment as they can survive on dry surfaces, in antiseptic solution and distilled water for many days. They can easily have transmitted to human body by sources like indwelling intravascular catheters, drain tubes from surgical site, surgical intervention and from other inanimate objects like bed rails, bedside tables, ventilators, air humidifiers and sinks and from these the NFGNB is transmitted to the patients.

In this study a total of 2021 blood samples were processed. In this study, overall incidence of bloodstream infection by NFGNB was based on significant bacterial growth in the blood cultures obtained from suspected patients was $732.7 \%$. Comparatively, in 2013 a study done in Eastern India had revealed 201 nonfermenters were isolated from 1650 clinical samples, accounting for an isolation rate from blood culture is $16.41 \%$. ${ }^{14}$ Where as another study in Gujrat by Patel et al., isolated 2397 (23.93\%) NFGNB, out of total 20721 various clinical samples, accounting for isolation rate of blood culture is $6.96 \%$. $^{15}$

Infection due to NFGNB can occur at any age. Bloodstream infections by NFGNB varied significantly within age groups, where the highest prevalence was recorded among patients at the 41 to 50 . Similarly, only few studies suggest a correlation between the infection due to NFGNB and age. A study, done in Eastern part of India in 2013 revealed that majority of the patients $(45 \%)$ were adults and above 45 years, which is similar to this current study. ${ }^{14}$

The highest number of isolates were Acinetobacter boumannii, comprises $27.69 \%$. Acinetobacter boumannii has emerged as an important opportunistic pathogen in healthcare systems. As it hard to desiccate, so difficult to eradicate and has numerous intrinsic and acquired mechanisms of drug resistance. Thus this organism possesses a great threat to the clinician as well as to microbiologists. These organisms found extensively in nature and are able to alive in environment. They can stay alive within disinfectants and can create problem in health care facilities spreading by cross contamination and causing to blood stream infections. $^{16}$

Strenotrophomonas maltophilia was the second common isolates (21.53\%). Stenotrophomonas maltophilia is water borne organisms and recently emerged as an important opportunistic pathogen in debilitated host. They are enraging as a known cause of infection in the nosocomial settings.

The isolates of this emerging pathogen from blood is quite difficult to interpret as primary pathogen. However if this isolate yields from a site which is supposed to be sterile, such as from blood, drain tip or CVP tip, then this isolate represents as true or primary pathogen. Muder et al., report same kind of study where he was reported a series of 91 patients with Stenotrophomonas maltophilia bacteraemia, among them 56\% did not reveal any clinically apparent portal of entry but $84 \%$ of these individuals had central venous catheter in place. ${ }^{17}$ In 2007 Gautam et al., isolated 22 Stenotrophomonas maltophilia. Out of which 13 were from the blood samples of bacteraemia patients and 9 were from respiratory isolates. ${ }^{18}$

In this study, Pseudomonas spp was another common organism causing BSIs. Pseudomonas are ubiquitous in nature as saprophytes. Earlier it is believed to be non pathogenic. But recently they have emerged as primary opportunistic pathogens in hospitalized patients as well as immunocompromised patients and 
responsible for causing variant infections including BSIs.They are very hard to desiccate, difficult to eradicate and has numerous intrinsic and acquired mechanisms of drug resistance. They can stay alive within disinfectants and can create problem in health care facilities spreading by cross contamination. The abuse and the unjudicial practice of antibiotics are responsible for the burgeoning resistance of commonly used antibiotics towards Pseudomonas. More over the multidrug resistance among these organisms makes the treatment of this infection difficult and expensive. ${ }^{19}$

Burkholderia cepacia complex (BCC) found in many niches of both natural and clinical environments BCC is emerging as an important cause of morbidity and mortality in hospitalized patients because of high intrinsic antibiotic resistance, such as aminoglycosides, chloramphenicol and polymyxins. An upsure of septicaemia due to $\mathrm{BCC}$ is documented in various studies. ${ }^{18}$

In our study from 65 NFGNBs we have isolated 3 isolates of B.cepacia and one isolate of Burkholderia multivorans from the blood taken in BACT/ALERT 3D SYSTEM bottle. The patients was diagnosed with sepsis and admitted in the ICU and the central venous line was in situ. Similarly, in 20062007 Gautam et al., isolated 39 isolates of BCC from various specimens. Out of these 39 total isolates, 30 isolates of $\mathrm{BCC}$ were obtained from 8601 blood cultures, accounting for $0.35 \%{ }^{18}$

In this current study we have yielded 2 isolates of Sphingomonas paucimobilis from blood samples. These isolates were obtained from the blood cultures of two young patients who were admitted in ICU and female medical ward for a long tenure with the diagnosis of septicaemia. We have isolated only one isolates of Moraxella group from the the central venous tip of a young female, admitted in ICU with the diagnosis of septicaemia.

The risk factors associated with this pathogen are intensive care admission, prolonged hospitalization, on mechanical ventilation, presence of central venous catheter, indwelling catheters, orthopaedic implants, unjudicial use of broad spectrum, antibiotics and comorbid conditions. These predisposing factors accelerate the occurrence of the blood stream infection due to these organisms.

These NFGNB are posing a great threat to human race as they are resistant to routinely used antibiotics. The abuse and the unjudicial practice of antibiotics are responsible for the burgeoning resistance of commonly used antibiotics towards NFGNB. The resistance to antimicrobials is increasing in recent years and almost resistance to all commonly used antibiotics. More over the multidrug resistance among these organisms makes the treatment of this infection caused by NFGNB difficult and expensive.

Pseudomonas aeruginosa shows a good sensitivity to Imipenem $(86.77 \%)$ which is almost similar to the study by Patel et al., who reported $94 \%$ sensitivity to this drug. ${ }^{15} \mathrm{~A}$ study by Rit et al., reported that P.aeruginosa were highly susceptible to Colistin (100\%), Imipenem (91.8\%) and Amikacin (69.3\%). ${ }^{14}$ In my study similarly Colisti (100\%), Imipenem (86.77\%) and Amikacin (64.02\%) revealed the same findings. The isolates of $P$. aeruginosa were sensitive to and Ciprofloacin (57.67\%), in comparison to this study another study by Patel et al., revealed a very low susceptibility rate to Amikacin (39.6\%) and Ciprofloacin (16.53\%). ${ }^{15}$ Here we found a good sensitivity to Gentamicin (57.14\%) unlike this current study, Rit et al., reported only $23.76 \%$ of susceptibility to Gentamicin. ${ }^{14}$ In this study $61.37 \%$ was 
susceptible to Piperacillin, similarly a study by Juyal et al., revealed $52.13 \%$ sensitive to this drug. ${ }^{20}$ Ciprofloacin and Ceftazidime both accounting for $57.67 \%$. Unlikely, a study by Patel et al., who reported only $24.6 \%$ susceptibility rate to Ceftazidime. ${ }^{15}$ Where as Carbenicillin (44.44\%) and Ceftazidimetazobactam $(17.98 \%)$ reveals quite a low sensitivity to this organism. In comparison to my study by Juyal et al., revealed $69.15 \%$ sensitivity to Piperacillin-tazobactam. ${ }^{20}$

Imipenem (88\%) show the highest sensitivity to Pseudomonas fluroscence, similarly a study by Rit et al., reported $100 \%$ sensitivity to Imipenem. ${ }^{14}$ In this current study Amikacin and Ceftazidime each of them show $66.7 \%$ sensitivity to Pseudomonas fluroscence. Similar to this study, Rit et al., revealed $66.66 \%$ sensitivity to Amikacin. However Rit et al., revealed a low sensitivity rate to Gentamicin (33.33\%) and Ciprofloxacin $(33.33 \%)^{14}$, where as in this study Gentamicin and Ciprofloxacin accounting for $71.4 \%$ and $61.9 \%$ susceptibility. Here Piperacillin accounting for $(71.4 \%)$.

Imipenem (89.65\%) shows the highest sensitivity for Pseudomonas putida, almost similar to this study, a study by Patel et al., revealed $100 \%$ susceptibility to Imipenem. ${ }^{15}$ The other isolates of P.putida show a moderate susceptibility pattern towards Amikacin (68.96\%) and Ciprofloxacin $(62.06 \%)$, where as in comparison to them study by Patel et al., revealed $100 \%$ sensitivity to Amikacine and Cefipime. ${ }^{15}$ Gentamicin show 58.62\%, Ciprofloxacin, Ceftazidime and Piperacillin an reveal a susceptibility rate of, $62.1 \% 55.17 \%$ and $51.72 \%$ respectively.

Imipenem (95.23\%) shows the highest sensitivity for Pseudomonas stutzeri, Gentamicin reveals $90.47 \%$. Ceftazidime shows $85.71 \%$, Ciprofloxacin and Amikacin reveal $80.95 \%$ individually.
The isolates of A.boumannii showed $60 \%$ were sensitive to Imipenem. Almost similar susceptibility of Imipenem (68.06\%) was reported by Juyal et al., ${ }^{20}$ In comparison to this Rit reported a good sensitivity to Imipenem $(90 \%) .{ }^{14}$ Where as another study by Parimal et al., revealed $72.9 \%$ sensitivity to Imipenem. ${ }^{15}$ In this study we have reported $52.2 \%$ susceptibility to Chloramphenicol and $48.9 \%$ to Gentamicin. In contrast to this study, another study by Rit revealed low susceptibility to Chloramphenicol $(28 \%)$ and Gentamicin (24\%). ${ }^{14}$ Where as Juyal et al., reported exactly similar sensitivity to Gentamicin $(48.61 \%){ }^{20}$ Close to it, in this study Amikacin and Norfloxacin each comprises of $47.8 \%$, in comparison to this study Rit et al., reported $62 \%$ susceptibility to Amikacin. ${ }^{14}$ In comparison to this Parimal et al., revealed a low sensitivity to Amikacin $(38.8 \%){ }^{15}$ However Juyal et al., reported a good sensitivity to Amikacin, accounting for $73.61 \% .^{20}$ In this study Ceftazidime shows a bit low sensitivity pattern, accounting for 37.8 $\%$.Similarly a study by Rit K, reported $28 \%$ sensitivity to Ceftazidime. ${ }^{14}$

Strenotrophomonas maltophilia revealed $100 \%$ sensitivity to Colistin, followed by Ertepenam 96.65\%, next to it was Ceftazidime and Ofloxacin, both accounting for $94.12 \%$, Ciprofloxacin (88.23\%), Gentamicin (84.12\%). Whereas, a study by Juyal et al., reported only $16.67 \%$ susceptibility to Gentamicin and almost resistant to Ceftazidime. ${ }^{20}$ However another study by Rit et al., reported a good susceptibility to Ceftazidime $(66.7 \%)^{14}$ In this study $70.58 \%$ susceptibility rate was for Cefotaxim. In this current study Amikacin and Imipenem reveal a good sensitivity, accounting for $72.36 \%$ and $79.71 \%$ sensitivity respectively, where as a study done by Juyal et al., reported almost resistant to Imipenem and Amikacin (33.33\%). ${ }^{20}$ 
Resistance to antimicrobials is common and has increased over the years among NFGNBs. Multidrug resistance among these organisms makes the treatment of infections caused by them, difficult and expensive. A large scale use of the third- generation Cephalosporins like Cefotaxime, Ceftriaxone, and Ceftazidime has led to the evolution of newer betalactamases such as the ESBLs. ESBLs are plasmid mediated enzymes that hydrolyze the oxyimino $\beta$ lactams and the Monobactams (Aztreonam) but have no effect on the Cephamycins (Cefoxitin, Cefotetan) and the Carbapenems (Imipenem). ${ }^{21,} 22,23$ Being plasmid mediated, they can be easily transferred from one organism to another. ${ }^{23} \mathrm{~A}$ rapid detection of ESBL and MBL positive isolates is necessary to control infection and to prevent their dissemination. In this study we have performed Combined Disc Diffusion Test (CDDT) to However in this study among the total 65 isolates of NFGNB, 20 isolates $(30.77 \%)$ were multidrug resistance (MDR). However, amidst these 20 isolates 11 (55\%) were ESBL- producers and rest (45\%) were MBL-producers. Among the total 65 isolates of NFGNB, 20 isolates $(30.77 \%)$ were multidrug resistance (MDR). However, amidst these 20 isolates $11(55 \%)$ were ESBL producers and rest (45\%) were MBLproducers.

In conclusion a large number of NFGNB are isolated as primary pathogen, which has a potential to cause BSIs. The remarkable thing about all these isolates is that these isolates obtained from the typical cases of HAI. These organisms have possibly come from inanimate objects like ventilator, humidifier, wash basin and from diluted disinfections. Most of the patients had high risk factors, like comorbid conditions (DM, hypertension, post renal dialysis, cardiac disease, tuberculosis etc) prolonged hospitalization, indwelling catheters and orthopaedics implants in situ and unjudicial use of antibiotics. Although
BSI rates declined over time, but BSI had high mortality and these NFGNB pathogens exhibited substantial antimicrobial resistance. Most effective antibiotics are colistin, imipenem, ertepenam, amikacin and gentamicin. There is a wide spread variability of antibiotic profile in common hospital for these pathogens. the antibiotic susceptibility can change from hospital to hospital set up and there may be a gross geographical variation.So, it is imperative that every hospital should monitor a proper antibiogram profile for these isolates from time to time to serve as a basic empirical therapy to prevent the development of MDR cases. Treating these pathogen should be based on the laboratory data after identifying the proper causative agents and antibiotic susceptibility result. Minimized the use and abuse of antimicrobial agents, proper surveillance of antibiotic panel, strict infection control measures and even simple yet proper hand washing method and by using disinfections of inanimate objects, can prevent the emergence Acinetobacter and can reduce the rate of MDR strains.

\section{References}

1. Govan J.R.W. Pseudomonas, Strenotrophomonas, Burkholderia. In: Collee J.G., Fraser A.G., Marmion B.P., Simmons A, editors. Mackie \& McCartney. Practical Medical Microbiology. $14^{\text {th }}$ ed. Churchill Livingstone; 1996. 413-23.

2. Winn W Jr, Allen S, Janda W, Koneman E, Procop G, Schreckenberger P, et al., The Nonfermentative Gram-Negative Bacilli. Koneman's Color Atlas and Textbook of Diagnostic Microbiology, 2006; 305-91.

3. Steinberg JP, Rio DC, Gram negative and Gram variable bacilli. Principles and Practice of Infectious diseases, 2005; 2: 2751-68.

4. Gales AC, Jones RN, Forward KR, Linaes 
J, Sader HS, Verhoef J, Emerging importance of multidrug -resistant Acinetobacter species and Stenotrophomonas maltophilia as pathogens in seriously ill patients: genetic patterns, Epidemiological features and trends in the SENTRY antimicrobial surveillance program. Clinical Infectious Disease, 2001; 32: 104-13.

5. Gardner P, Griffin W.B, Smart MN, Kunz L J, Non-fermentative gram negative bacilli of nosocomial interest. American Journal of Medicine, 1970; 48: 735-48.

6. Parajuli N.P, Parajuli H, Pandit R, Shakya J, Khanal P.R, Evaluating the Trends of Bloodstream Infections among Pediatric and Adult Patients at a Teaching Hospital of Kathmandu, Nepal: Role of Drug Resistant Pathogens. Canadian Journal of Infectious Diseases and Medical Microbiology, volume 2017

7. C. Viscoli, "Bloodstream Infections: the peak of the iceberg," Virulence, vol. 7, no. 3, pp. 248-251, 2016.

8. K.B. Laupland, "Incidence of bloodstream infection: are view of population-based studies," Clinical Microbiology and Infection, vol. 19, no. 6, pp. 492-500, 2013.

9. R. S. Munford and A. F. Su redini, "Sepsis, severe sepsis, and septic shock," in Mandell, Douglas and Bennett's Principle and Practice of Infectious Diseases. Volume 1, G. L. Mandell, J. E. Ben- nett, and R. Dolin, Eds., pp. 9871010, Churchill Livingstone- Elsevier, 2010.

10. N. Obeng-Nkrumah, A.-K. Labi, N. O. Addison, J. E. M. Labi, and G. AwuahMensah, "Trends in paediatric and adult blood- stream infections at a Ghanaian referral hospital: a retrospective study," Annals of Clinical Microbiology and Antimicrobials, vol. 15, no. 1, article 49, 2016.
11. King E.O, Ward M.K, Raney DE. Two simple media for the demonstration of pyocyanin and fluorescein. Journal of Laboratory Clinical Medicine, 1954;44:301-7.

12. National Committee for Clinical Laboratory standards Methods for dilution antimicrobial susceptibility tests for bacterial that grow aerobically. Informational supplement M100-S23. National Committee for Clinical Laboratory Standards 2013.Wayne, Pa.

13. Chong Y., Lee K., Shin H., Kim Y.,Yong D., Yum J., Modified Hodge and EDTA disk synergy test to screen metallo $\beta$ lactamases producing strains of Pseudomonas and Acinetobacter species. Society of Clinical Microbiology. 2001;7(2):88-91.

14. Rit K, Nag F, Rar, H.J, Maity PK, Prevalence and Susceptibility Profiles of Nonfermentative Gram-negative Bacilli Infection in a Tertiary care hospital of Eastern India. Indian Journal of Clinical Practice.2013,451-5.

15. Patel P.H., Pethani J.D., Rathod S.D., Chauhan B., Shah P.D. Prevalence of nonfermenting Gram negative bacilli infection in tertiary care Hospital in Ahmedabad, Gujarat. Indian Journal of Basic and Applied Medical Research. 2013; 6(2):608-13.

16.Pathi B., Mishra S.N., Panigrahi K., Poddar N., Lenka P.R., Mallick B., Pattanik D., Jena J. Prevalence and antibiogram pattern of Pseudomonas aeruginosa in a tertiary care hospital from Odisha, India. Transworld Medical journal.2014; 1(3):77-80.

17. Muder R.R., HarrisA.P., Muller S., Edmond M., Chow J.W., Papadakis K., Wagener M.W., Bodey G.P., Steckelberg J.M. Bacteraemia due to Stenotrophomonas (Xanthomonas) maltophilia: a prospective multicenter study of 91 episodes. Journal of Clinical 
Infectious Disease.1996; 22: 508-12.

18. Gautam V, Ray P, Vandamme P, Chatterjee SS, DasA, Sharma K, Rana S, Garg RK, Madhup, Mahajan M, Sharma $\mathrm{M}$, Identification of lysine positive nonfermenting gram negative bacilli (Stenotrophomonas maltophilia and Burkholderia cepacia complex). Indian Journal of Medical Microbiology, 2009; 27(2): 128-33.

19. Edith B.H., Deborah A.H., Speert D.P., Pseudomonas. In: Baron EJ, Jorgensen JH. Landry ML, Pfaller MA (editors). Manual of Clinical Microbiology. $9^{\text {th }}$ ed. Washington DC: American Society for Microbiology; 2007. p.734-48.

20. Juyal D., Prakask R., ShanakarmarayanS. A., Sharma M., Negi V., Sharma N. prevalence of nonfermenting gram negative bacilli in vitro susceptibility pattern in a tertiary care hospital of Uttarakhand: A study from foothills of Himalayas. Soudi journal of Heath Sciences. 2013; 2(2):108-12.
21. Kaur A.,Singh S.Prevalence of Extended Spectrum Betalactamase (ESBL) and Metallobetalactamase (MBL) Producing Pseudomonas aeruginosa and Acinetobacter baumannii Isolated from Various Clinical Samples. Hindawi Journal of Pathogens. Volume 2018, Article ID 6845985, 7 pages https://doi.org/10.1155/2018/6845985

22 Z.A. Memish, A.M. Shibl, A.M. Kambal, Y.A. Ohaly, A. Ishaq, and D. M. Livermore, "Antimicrobial resistance among non- fermenting gram-negative bacteria in Saudi Arabia," Journal of Antimicrobial Chemotherapy, vol. 67, no. 7, Article ID dks091, pp. 1701-1705, 2012.

23. A. O. Okesola and A. A. Oni, "Occurrence of Extended-Spectrum Beta- Lactamase- Producing Pseudomonas aeruginosa Strains in South-West Nigeria," Research Journal of Medical Sciences, vol. 6, no. 3, pp. 93-96, 2012

\section{How to cite this article:}

Nabamita Chaudhury, Retina Paul, R.N. Misra, Sankha Subhra Chaudhuri, Shazad Mirza and Sukanta Sen. 2019. Evaluating the Trends of Bloodstream Infections by Nonfermenting Gram Negative Bacilli among the Patients in a Tertiary Care Hospital of Western Part of India and its Antibiogram. Int.J.Curr.Microbiol.App.Sci. 8(01): 1149-1162. doi: https://doi.org/10.20546/ijcmas.2019.801.121 